\title{
Complutum
}

ISSN: 1131-6993

\section{El abrigo de Hoya de Navarejos III (Tormón, Teruel). Nuevas perspectivas para el análisis del arte levantino interior ${ }^{1}$}

\author{
Manuel Bea Martínez²
}

Recibido: 25 de noviembre de 2016 / Aceptado: 26 de julio de 2017

Resumen. Se presenta un nuevo conjunto de arte levantino, denominado Hoya de Navarejos III, localizado en plena Sierra de Albarracín. Tanto sus representaciones rupestres como su especial emplazamiento, en el interior de una cámara natural asociada a un recinto exterior delimitado por lajas hincadas, hace que se pueda calificar como una de las estaciones rupestres más destacadas y singulares del arte levantino de la Península Ibérica. Estas características, junto a las de otros conjuntos recientemente descubiertos o estudiados, hacen que la clasificación levantina de sus figuraciones en relación al contexto referido invite a replantear determinados aspectos definitorios del propio arte levantino, atendiendo tanto a cuestiones estilísticas como de filiación crono-cultural.

Palabras clave: Arte levantino; terminología; Sierra de Albarracín; santuario rupestre; pintura blanca; lajas hincadas.

\section{[en] The Rock art Shelter of Hoya de Navarejos III (Tormón, Teruel). New Perspectives for the Analysis of the Inner Levantine Rock Art}

\begin{abstract}
A new Levantine rock-art shelter, named Hoya de Navarejos III, recently found I the mountain range of Albarracín (Teruel) is presented. Because of its motifs and its special location in the inner part of a natural chamber associated with an external enclosure bounded by thrust vertical slabs. These elements mean that this rock-art group can be described as one of the most interesting and singular sites of the Iberian Peninsula. The Levantine classification of the motifs in relation to the aforementioned context invites us to rethink certain aspects about the definition of the Levantine rock-art itself, concerning both to stylistic and chrono-cultural affiliation issues.

Keywords: Levantine Rock Art; Terminology; Albarracín Mountain range; rock-art sanctuary; White pigment; Thrust slabs.
\end{abstract}

Sumario. 1. Introducción. 2. Descripción. 2.1. El Conjunto decorado. 3. Espacio simbólico. 4. Estilo, temática y cromatismo. 5. Sobre terminologías, clasificaciones y ordenaciones. 6. Conclusiones.

Cómo citar: Bea Martínez, M. (2017): El abrigo de Hoya de Navarejos III (Tormón, Teruel). Nuevas perspectivas para el análisis del arte levantino interior. Complutum, 28(1): 37-50

\section{Introducción}

El término municipal de Tormón (Teruel) forma parte, junto a los núcleos de Bezas y Albarracín, de una de las áreas geográficas con arte rupestre postpaleolítico más singulares de la fachada oriental de la Península Ibérica. De los 23 conjuntos rupestres de la Sierra de
Albarracín declarados Patrimonio Mundial, cuatro se localizan en la denominada Casa Forestal de Tormón: Ceja de Piezarrodilla (Obermaier 1927; Obermaier y Breuil 1927; Beltrán 1968: 148; Piñón 1981a; 1982: 148151), Cerrada del Tío Jorge (Obermaier 1927; Obermaier y Breuil 1927; Almagro 1970: 360; Beltrán 1968: 147; Piñón 1982: 144-147), Ca-

\footnotetext{
1 Programa “Torres Quevedo" (Ministerio de Economía y Competitividad), 3D Scanner Patrimonio e Industria. Spin-off Universidad de Zaragoza. Grupo "Primeros Pobladores del Valle del Ebro" (H-07).

2 Universidad de Zaragoza, Área de Prehistoria, Departamento de Ciencias de la Antigüedad (España)

E-mail: manubea@unizar.es
} 
bras Blancas (Bader et al. 1981; Collado et al. 1991-1992; Beltrán 1993: 160) y Paridera de Tormón (Beltrán 1997). Pero junto a éstos, y desde 2008, se han venido realizando hallazgos de abrigos decorados, algunos de ellos singulares, que convierten a la zona en una de las áreas con arte rupestre más destacadas de todo Aragón, contando ya en el núcleo de Tormón con 20 conjuntos rupestres (incluido el abrigo del Prado de las Olivanas, geográficamente cercano al núcleo de Tormón, a pesar de que administrativamente forme parte de la villa de Albarracín), 18 de ellos con representaciones levantinas (Bea 2014).

La estación de Hoya de Navarejos III, descubierta de forma casual por R. Canet en 2008, se localiza a 1,5 kilómetros del núcleo rupestre de la Casa Forestal. Las características geológicas, geomorfológicas y de cubierta vegetal de la zona son plenamente coincidentes con las observadas para la Sierra de Albarracín, formando parte del entorno protegido del Rodeno, un tipo de arenisca definida dentro de la facies Buntsandstein (final del Paleozoicoinicios del Mesozoico).

El conjunto rupestre se ubica en una formación de arenisca, cuyas particularidades com-

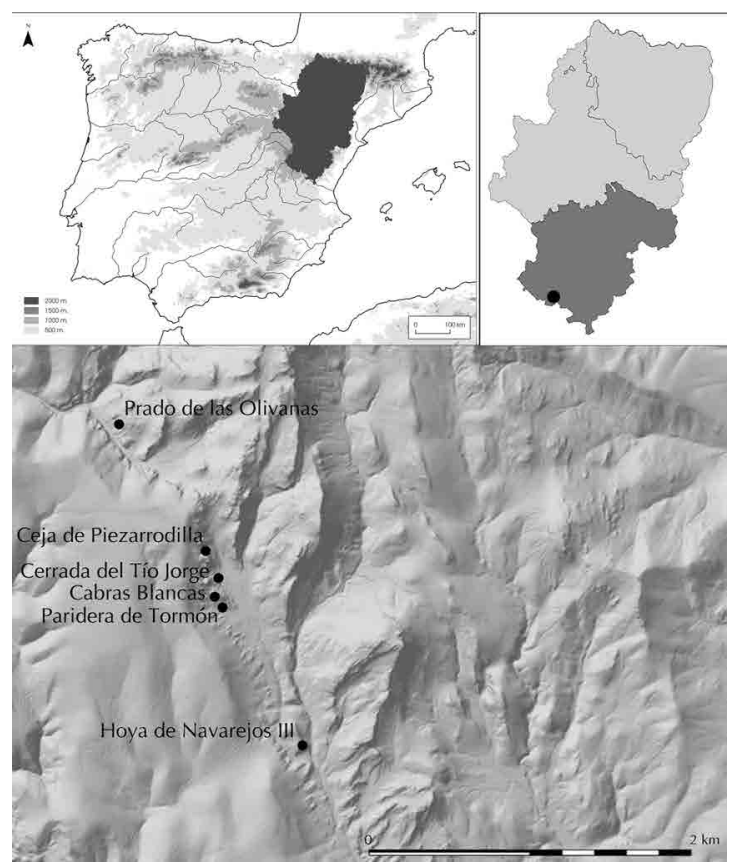

Figura 1. Localización del abrigo de Hoya de Navarejos III. positivas determinan una gran vulnerabilidad a los procesos erosivos. La meteorización de la roca confiere al relieve modelados muy característicos con formas de tormos o torres, taffonis, callejones... generando en muchas ocasiones abrigos abiertos al aire libre, algunos de los cuales serían aprovechados para realizar los conjuntos pictóricos.

\section{Descripción}

El caso que nos ocupa es singular. El abrigo se abre en lo alto de una formación arenisca de suave morfología. Los procesos de alteración naturales determinaron la ampliación de fracturas en el soporte que culminaría con la individualización de bloques. Uno de estos bloques se desplazó de la zona basal de la formación rocosa a partir de una línea de fractura, adoptando una disposición transversal al pie mismo de la roca base. El desplome lateral y el leve desplazamiento posterior del bloque conformó un espacio interior de reducidas dimensiones $(273 \mathrm{~cm}$ de longitud, $79 \mathrm{~cm}$ de anchura y $141 \mathrm{~cm}$ de altura máxima) y desarrollo paralelo a la roca base. En el interior del mismo se realizaron los dos paneles con pinturas rupestres (Fig. 2).

Resulta evidente una búsqueda deliberada, sin que podamos precisar el momento en que se llevara a cabo, de aislar completamente del interior de la cámara, de manera que se emplearon pequeños bloques y lajas de piedra arenisca para cerrar la pequeña fisura existente entre la parte superior del bloque desprendido y la formación matriz.

En el interior de la cámara se observan abundantes bloques de piedra, desprendidos del cierre artificial de la parte superior de la pared Sur, así como fragmentos de lajas, algunos de los cuales podrían haber formado parte del cierre de la entrada a la cámara. El acceso resulta perfectamente practicable, advirtiéndose restos de una laja hincada transversal a la boca, a modo de cierre, que subraya el carácter de aislamiento del interior.

El espacio exterior también puede ser interpretado como un elemento conformador del espacio simbólico rupestre, al haberse delimitado mediante lajas hincadas una superficie de unos $30 \mathrm{~m}^{2}$, en cuyo interior queda el acceso al conjunto rupestre (Fig. 3). En una prospección visual del espacio referido no se localizó elemento material alguno en superficie. 


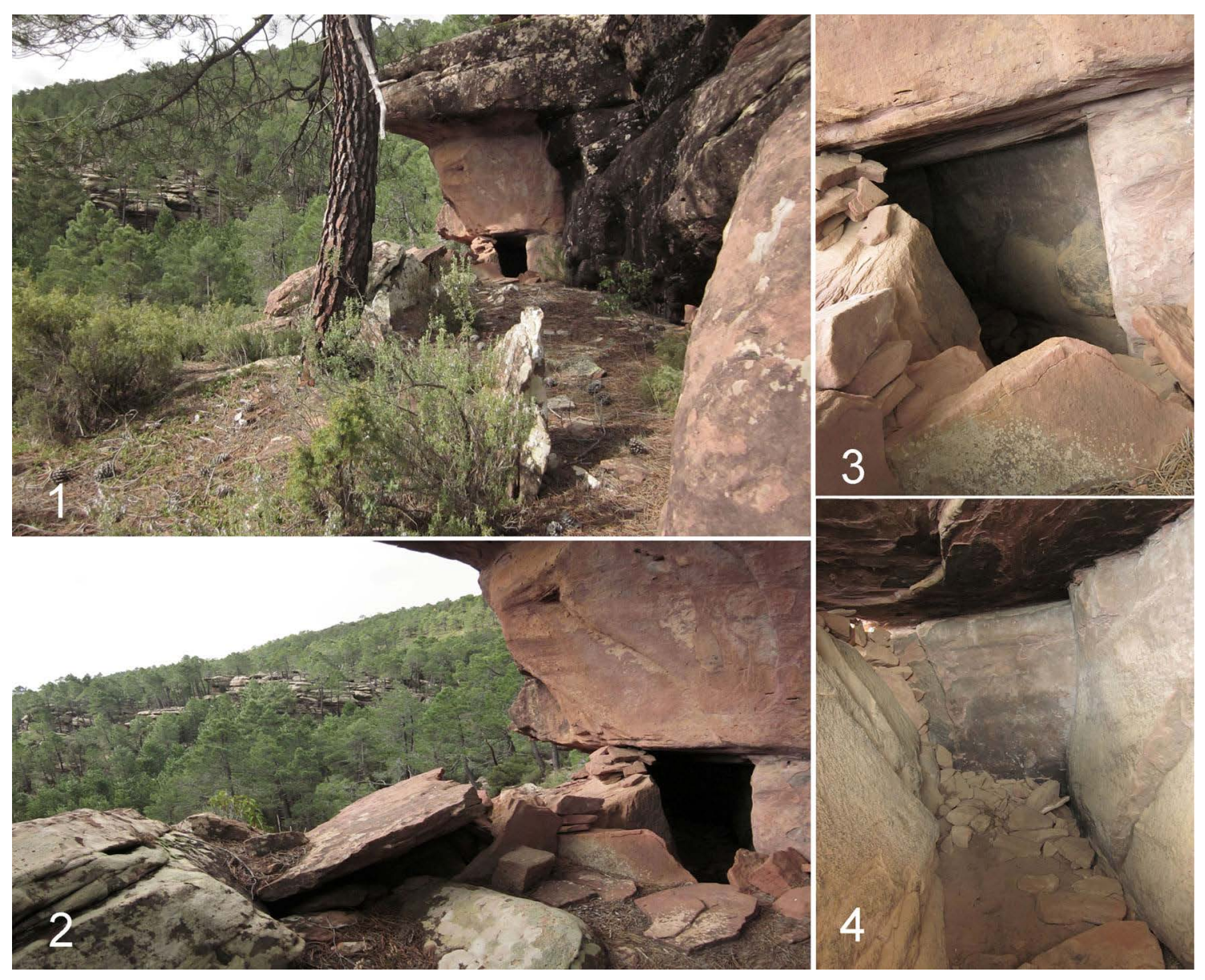

Figura 2. 1. Vista general del conjunto de Hoya de Navarejos III. Se aprecia la disposición de las lajas hincadas delimitando un espacio exterior. Al fondo la abertura que da acceso a la cámara con los paneles decorados. 2. Zona inmediata a la boca de entrada a la cámara. Se aprecia la disposición de algunas lajas hincadas para tapar la entrada. 3. Detalle de la entrada a la cámara. En la pared interior se puede apreciar el motivo 1 del panel 1. 4. Interior de la cámara, la pared del fondo contiene el panel 2.

\subsection{El Conjunto decorado}

En el interior de la estancia se documentan dos paneles decorados bien diferenciados, uno cercano a la boca de la cámara (panel 1) y otro en el fondo de la misma (panel 2).

\section{Panel 1}

La pared Norte de la cámara cuenta con 246 $\mathrm{cm}$ de desarrollo lineal y una media de $127 \mathrm{~cm}$ de altura. A $74 \mathrm{~cm}$ de la entrada, bien visible desde el exterior, se localiza el panel 1 compuesto por tres motivos, todos ellos toros de color blanco. La totalidad de los motivos se encuentran en la mitad superior de la pared, el más bajo de ellos a $70 \mathrm{~cm}$ de altura respecto al suelo actual (Fig. 4).
Motivo 1. Cabeza de bóvido orientada a la derecha. Se localiza a $74 \mathrm{~cm}$ de la entrada y a $114 \mathrm{~cm}$ de altura respecto al suelo. Destacan los cuernos en media luna.

Dimensiones: $11,8 \mathrm{~cm}$ de longitud, $11 \mathrm{~cm}$ de altura. Color: blanco.

Motivo 2. Gran bóvido orientado a la derecha. Presenta un estado de conservación relativamente bueno, aunque bastante afectado por la acumulación de polvo, se aprecia la práctica totalidad de la figura.

Las proporciones del toro no resultan correctas, presentado una hipertrofia del cuerpo (muy alargado) con respecto a la longitud de las patas, excesivamente cortas, un patrón morfoestilístico común en las figuraciones de bóvidos de la Sierra de Albarracín. 


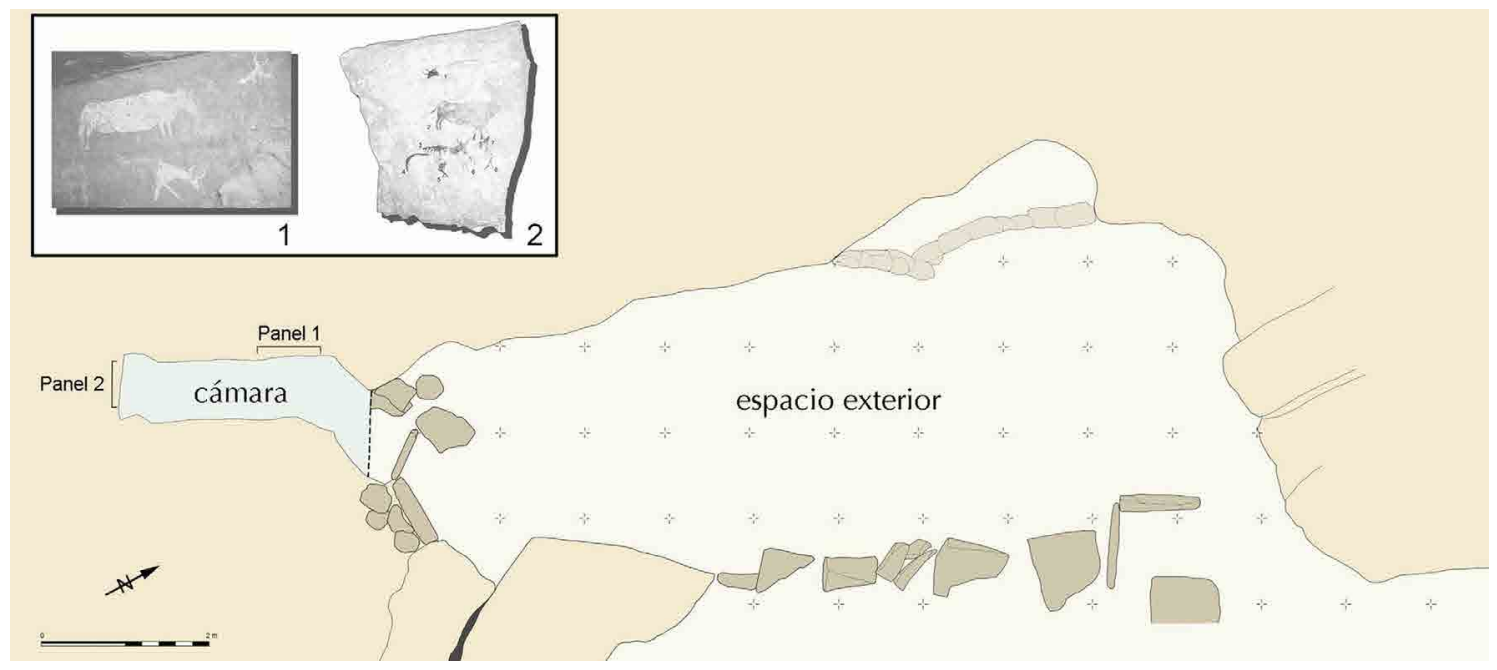

Figura 3. Planta del yacimiento en la que se indica la disposición de la cámara decorada con respecto al especio exterior delimitado mediante lajas.
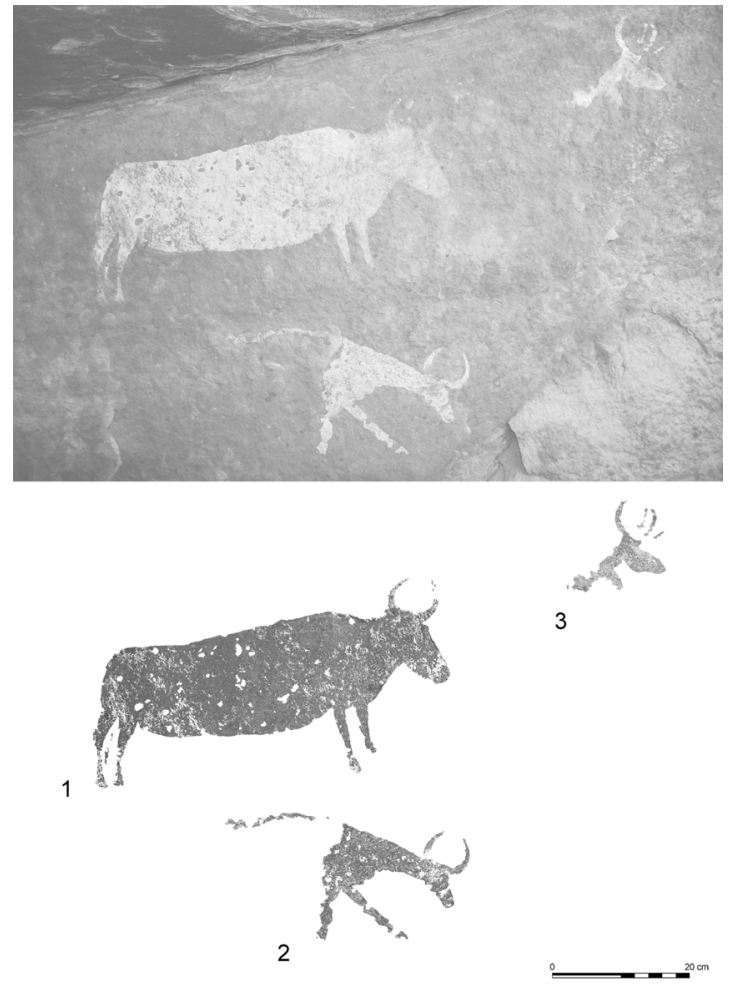

Figura 4. Calco del panel 1 de Hoya de Navarejos III.

Como en el anterior caso, los cuernos fueron representados en perspectiva frontal, en forma de media luna.

Dimensiones: 48,4 cm de longitud, 19,5 cm de altura. Color: blanco.
Motivo 3. Mitad delantera de un toro de grandes dimensiones. A $12 \mathrm{~cm}$ por debajo del motivo 2.

Se conserva parte del cuello y la cabeza, con los cuernos de media luna. Fue representado en actitud dinámica tranquila, con las patas delanteras abiertas en ángulo agudo y el cuello largo e inclinado hacia abajo.

Dimensiones: $15,5 \mathrm{~cm}$ de longitud. Color: blanco.

\section{Panel 2}

Se dispone en la pared final de la estancia, la que cierra el espacio en su lado Este, con unas dimensiones de $87 \mathrm{~cm}$ de anchura en la parte alta, $70 \mathrm{~cm}$ de anchura en la baja y 112 $\mathrm{cm}$ de altura máxima. Se encuentra bastante oscurecida, quizá afectada por ahumados o por efecto de la acción biológica o microbiológica. Esta circunstancia, unida a la menor llegada de luz al lugar, dificulta la correcta lectura del panel, de manera que la identificación y definición de algunos de los restos pictóricos resulta complicada.

A pesar de la dificultad de la observación por la mala conservación global del conjunto, una primera aproximación arroja la existencia de, al menos, tres bóvidos, un cuadrúpedo indeterminado, un posible antropomorfo y otros restos inidentificables (Fig. 5).

Al contrario de lo observado en el primer panel, en el que nos ocupa, las figuras son todas de tonalidades oscuras, gris o negras. 
Motivo 1. Prótomo de bóvido orientado a la izquierda. Se localiza en la parte alta del panel decorado. Fue representado con la cabeza agachada, en actitud de embestida. Los cuernos, de trazo fino, fueron representados en perspectiva semitorcida. La cabeza tiene forma triangular, reducida con respecto a grosor del cuello conservado, y cuenta con cierto tratamiento detallado del morro y boca.

Dimensiones: $8,5 \mathrm{~cm}$ de longitud, $4 \mathrm{~cm}$ de anchura del cuello. Color: Gris.

Motivo 2. Cuerpo de un gran bóvido orientado a la derecha. No se conserva la cabeza del animal. Destacan las buenas proporciones corporales del animal, así como la rotundidad y potencia de sus formas, con indicación de la joroba y pecho. Muestra diversos rasgos naturalistas y detalles anatómicos como la cola o el corvejón en las patas traseras. Las delanteras se representaron abiertas en ángulo agudo.
Dimensiones: $23,5 \mathrm{~cm}$ de longitud (en lo conservado), $17,3 \mathrm{~cm}$ de altura. Color: Negruzco.

Motivo 3. Bóvido orientado a la derecha y en color negro. Destaca el tratamiento muy naturalista de la cabeza y de los cuernos, con una morfología y proporciones de la cabeza, morro, cuello y joroba muy realistas. El cuerpo, reconocible sólo la parte superior del animal, fue representado mediante poco más que un perfilado. De éste parten una serie de trazos lineales, finos y paralelos entre sí y de tamaños progresivamente descendentes hacia la parte trasera del toro. Estos trazos recorrerían el interior del cuerpo del animal, quizá pretendiendo representar las costillas.

Se podría apreciar indicios de la existencia de un repintado en este motivo. Entre los finos y largos cuernos del animal se aprecian restos, muy mal conservados, de lo que podría inter-
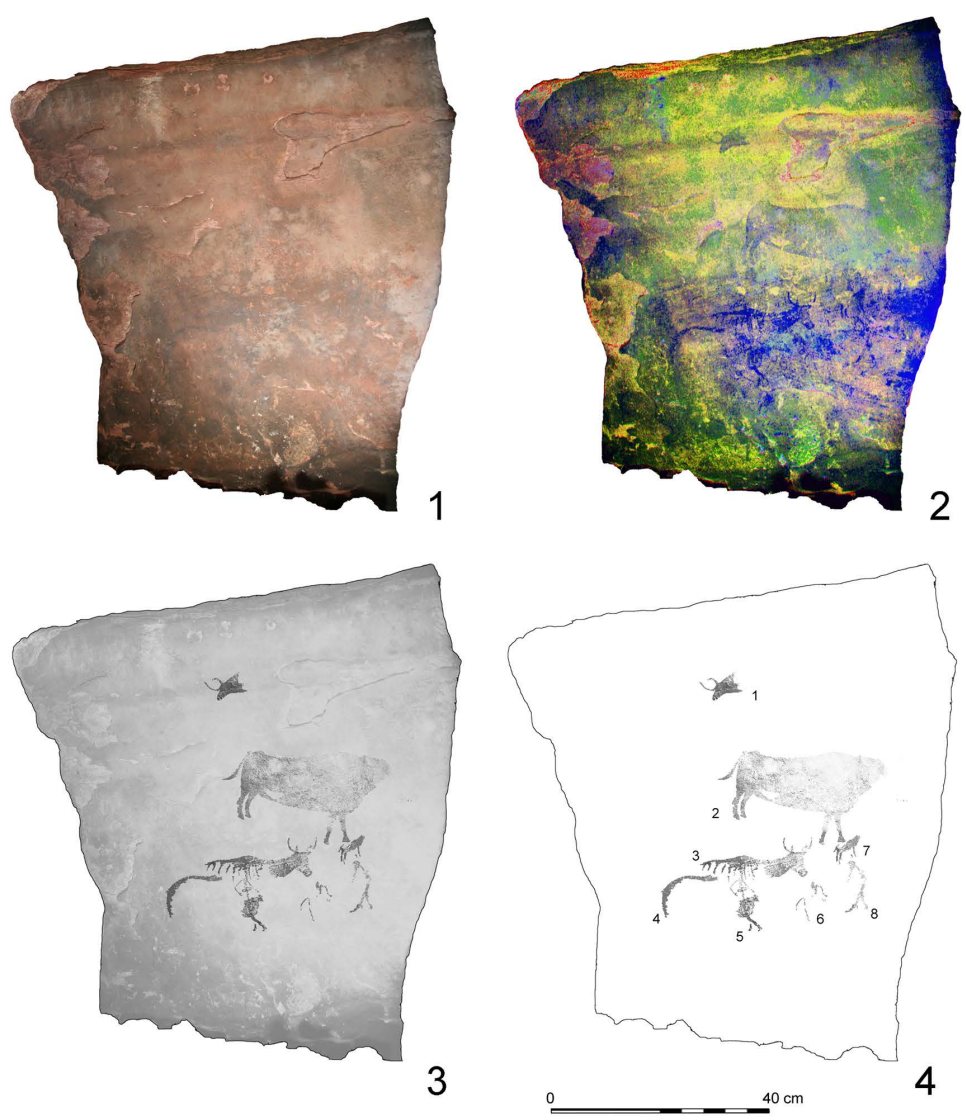

Figura 5. 1. Fotografía del panel 2 de Hoya de Navarejos III; 2. Fotografía con tratamiento digital de imagen; 3 y 4. Calcos del panel 2. 
pretarse como otra cornamenta subyacente al motivo descrito.

Dimensiones: $24,3 \mathrm{~cm}$ de longitud. Color: negro.

Motivo 4. Elemento lineal de morfología curva. Se encuentra inmediatamente debajo del motivo 3, en la parte izquierda. Se define como un simple trazo inacabado, cuya forma podría recordar a la grupa de un cuadrúpedo sin que se pueda precisar su verdadera naturaleza.

Dimensiones: $10,16 \mathrm{~cm}$ de longitud máxima $1,44 \mathrm{~cm}$ grosor máximo del trazo. Color: Gris.

Motivo 5. Posible antropomorfo. Una serie de restos de trazos y manchas se combinan de manera que no resulta posible realizar una lectura concluyente. Se advierten una serie de trazos lineales, dos en la zona alta ligeramente curvados y en relación con otro trazo lineal de tendencia vertical. Casi a mitad de desarrollo de este trazo se aprecia otro corte, más grueso y horizontal que lo cruza. El elemento lineal vertical parece tener continuidad a través de una mancha de color informe. Por debajo de ésta se observa un nuevo trazo lineal inclinado hacia la derecha y otro más corto aislado a la izquierda de éste.

Sin poder realizar una afirmación definitiva, en conjunto, este motivo se podría corresponder con una figura antropomorfa orientada a la derecha y de rasgos muy estilizados, similares a los del motivo 8 .

Dimensiones: $10,5 \mathrm{~cm}$ de altura máxima. Color: Gris.

Motivo 6. Restos inidentificables de tendencia lineal.

Dimensiones: $0,33 \mathrm{~cm}$ de grosor del trazo. Color: Gris.

Motivo 7. Pequeño cuadrúpedo indeterminado. Se trata de la figura de un pequeño animal situado bajo la pata delantera del motivo 2 , sin que llegue a apreciarse superposición alguna. El zoomorfo se orienta a la derecha, en una disposición ligeramente inclinada hacia arriba. Son bien visibles las cuatro patas, una cola corta el cuerpo y el cuello, sin que se haya conservado o se llegara a representar la cabeza.

Dimensiones: $5,76 \mathrm{~cm}$ de longitud máxima. Color: Gris.

Motivo 8. Posible antropomorfo. Se trataría de un motivo humano muy estilizado orientado a la izquierda. El cuerpo aparecería arqueado hacia delante, con un engrosamiento en la parte final que podría corresponderse con la cabe- za. Del extremo superior parte un trazo lineal hacia abajo, que podría interpretarse como un brazo. Las piernas, sin concesiones naturalistas, aparecerían abiertas en ángulo agudo.

Dimensiones: $9,6 \mathrm{~cm}$ de altura máxima. Color: Gris.

En diferentes puntos de la superficie del panel 2 se han podido observar pequeñas manchas de morfología informe de color rojo, textura pastosa y aspecto graso. Sin que resulte posible identificar motivo alguno (figurativo o no) merece la pena destacar que manchas de características afines se han podido constatar recientemente en otros conjuntos de la serranía, como los de la Paridera de las Tajadas y Contiguo a la Paridera en Bezas (Bea y Angás 2013).

\section{Espacio simbólico}

La cavidad natural en la que se localizan los paneles decorados presenta una laja de piedra rota e hincada en las proximidades de la entrada y transversal a ésta, que debió de cerrar el espacio interior ${ }^{3}$. Asimismo, se observa en el exterior, un espacio delimitado por una serie de lajas hincadas en la tierra. Se establece así una clara división en dos espacios asociados, uno interior y otro exterior. En el primero de éstos, la ubicación de los paneles pintados aparece también como un elemento de singularidad dentro del arte levantino. Así, al margen de algún ejemplo con figuraciones de dudosa inclusión dentro del ciclo levantino, como el de Peña Rubia de Ceheguín (Beltrán 1987; Beltrán y San Nicolás 1988), resulta extraordinariamente singular encontrar motivos tradicionalmente definidos como levantinos en el interior de cavidades. En este sentido, tan sólo se puede citar el conjunto del Hocino de Chornas (Obón, Teruel), con motivos humanos próximos a los estandares levantinos $(\mathrm{Bu}-$ rillo y Picazo 1981). En otros algunos casos, en los que se constata la existencia de paneles levantinos en relación a abrigos más o menos profundos, como pueden ser los de El Milano (San Nicolás 2009), la decoración de tendencia naturalisa se presenta en las paredes exteriores $\mathrm{y}$, por lo tanto, bien iluminadas, o bien, como en Cova Roja III (Barciela et al. 2015), donde a pesar de que las pinturas levantinas se localizan en la zona profunda, las grandes dimensiones del abrigo hacen que el panel interior se encuentre perfectamente iluminado. 


\section{Estilo, temática y cromatismo}

Resultan evidentes las diferencias estilísticas, cromáticas y de dimensiones de los motivos así como de la propia ubicación de los paneles. El único elemento en común existente entre ambos es la temática: el toro confiere unidad al conjunto, aunque en gran medida también lo hace al núcleo rupestre de toda la Sierra de Albarracín (Prado del Navazo, Cocinilla del Obispo, Figuras Diversas, Medio Caballo, Arquero de los Callejones, Cerrada del Tío Jorge, Ceja de Piezarrodilla, Prado de las Olivanas, Arroyo de Bezas I) con una clara relación con los conjuntos de Cuenca (Peña del Escrito, Selva Pascuala).

Los paralelos más cercanos para los bovinos del panel 1 de Hoya de Navarejos III subrayan, precisamente, la importancia de esa unidad artística de la Sierra de Albarracín, compartiendo una misma concepción técnico-configurativa. Así, caben destacar las grandes figuras de toros de Ceja de Piezarrodilla; los toros del Prado de las Olivanas, Cocinilla del Obispo, Medio Caballo o los del Prado del Navazo. En este último caso, además, se presenta una disposición de los motivos muy similar, con un gran toro de cuerpo alargado, patas cortas, rectas y paralelas, cuello muy corto y astas en media luna (motivo 3 del Prado del Navazo, según Piñón 1982: 43); otro de menores dimensiones, a un nivel inferior, con las patas abiertas en ángulo agudo, cuello largo e inclinado: motivos 8 y 11 de Prado del Navazo (Piñón 1982: 43-44), los 14 y 15 del Prado de las Olivanas (Piñón 1982: 156) o el motivo 2 de la Ceja de Piezarrodilla; y un tercero con la testuz alzada, cuello bien definido y cabeza alargada con la plasmación de la quijada redondeada (motivo 6 de Prado del Navazo, según Piñón 1982: 43) (Fig. 6).

La tipología de las representaciones de bóvidos del panel 2 difiere sensiblemente de aquella observada para los ejemplares del primer panel. Tanto las dimensiones de los ejemplares como el color y la disposición de los motivos hacen factible su inclusión en una fase decorativa diferente. No obstante, los paralelos estilísticos se encuentran también cercanos. Así, para el motivo 1 del panel 2 encontramos un modelo prácticamente exacto en la Cerrada del Tío Jorge, ubicado en el prado de la Casa Forestal de Tormón. A pesar de que en Hoya de Navarejos III tan sólo se representó la cabeza del animal y que en la Cerrada del Tío Jorge se conserva prácticamente la mitad delantera del bóvido (Bea y Angás 2015), se observa una perfecta coincidencia en la morfología y actitud de las cabezas de ambas figuraciones.

El motivo 2 presenta unas formas contundentes, una figura robusta pero relativamente bien proporcionada, apreciándose un ligero alargamiento del cuerpo con respecto a la altura de las patas. Este mismo patrón, mucho más marcado en otros motivos, es típico de las figuraciones de bóvidos de Albarracín y Tormón.

El motivo 3 aparece, por el momento, como un unicum dentro del corpus figurativo levantino. En la Sierra de Albarracín encontramos otros motivos de dimensiones medias con un alto grado de naturalismo en la representación de la cabeza y con las astas en perspectiva torcida, como en Barranco de las Olivanas o en el panel 3 del Arquero de los Callejones Cerrados. Pero en ningún caso el tratamiento del cuerpo del animal obedece a lo representado en Hoya de Navarejos III. Es, además, junto al ejemplar de Ceja de Piezarrodilla, el único de toda la Sierra de Albarracín en el que se observan dos pares de cuernos.

El motivo 7, un zoomorfo de pequeñas dimensiones y acéfalo, no presenta unas características formales que permitan singularizarlo y clasificarlo en una etapa concreta del desarrollo artístico de la serranía de Albarracín. No aparece como una temática diferenciadora; sin embargo, encontramos algún ejemplo, siempre escasos, de pequeños animales de aspecto tosco en otros conjuntos de la Sierra tanto en color rojo como en blanco. Así se constata en los abrigos ya conocidos del abrigo del Ciervo, así como en el recientemente descubrimiento del Barranco de la Casa Forestal I. En el caso del abrigo del Ciervo, además, esta figuración animal comparte espacio (no es posible determinar si también escena) con un motivo humano muy estilizado, como los del abrigo de Navarejos III.

En esta línea, y para el caso de las posibles representaciones humanas (motivos 5 y 8), su análisis resulta algo más complejo debido a la dificultad en la lectura que presentan estas figuraciones, aspecto que determina su definición sólo como posibles antropomorfos. Sin embargo, atendiendo a su descripción como tales, los rasgos muy estilizados de las figuras (prácticamente esquemáticas) encuentran perfecto acomodo en las características generales para figuraciones humanas de la Sierra de Albarracín (Fig. 7). Los paralelos estilísticos para estos motivos muy estilizados, casi 

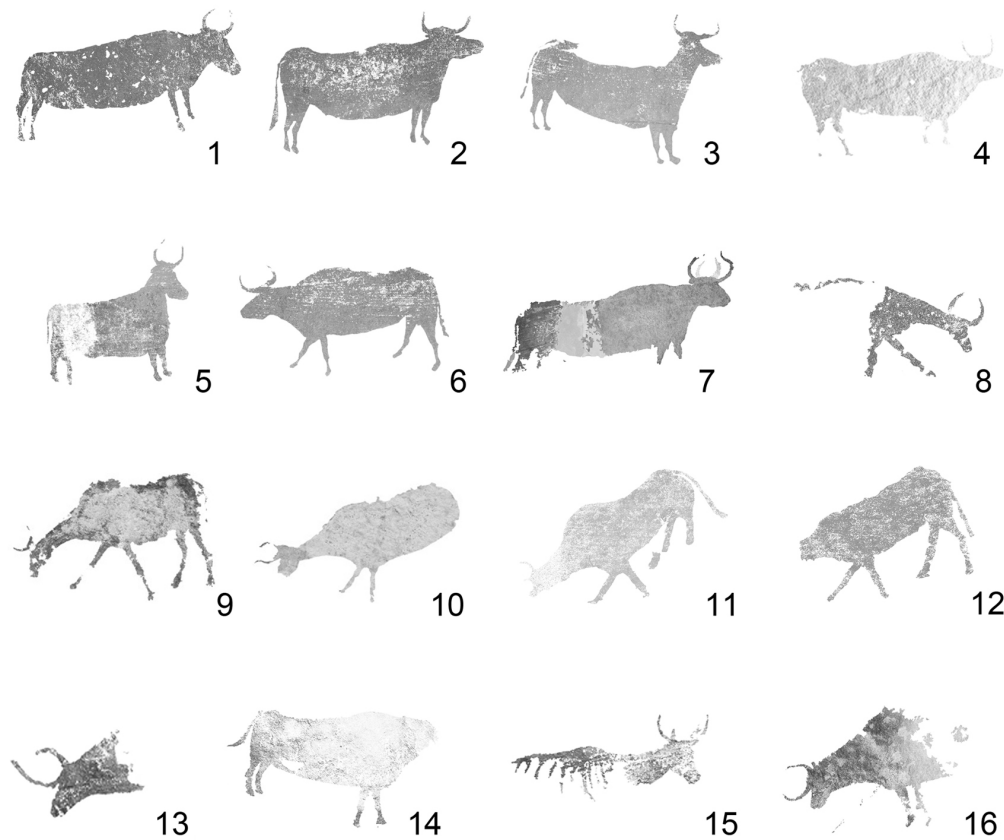

Figura 6. Paralelos para las representaciones de bóvidos. 1, 8, 13, 14 y 15. Hoya de Navarejos III; 2, 3, 5, 6, 11 y 12. Prado del Navazo; 4. Cocinilla del Obispo; 7. Ceja de Piezarrodilla; 9 y 10. Prado de las Olivanas; 16. Cerrada del Tío Jorge.
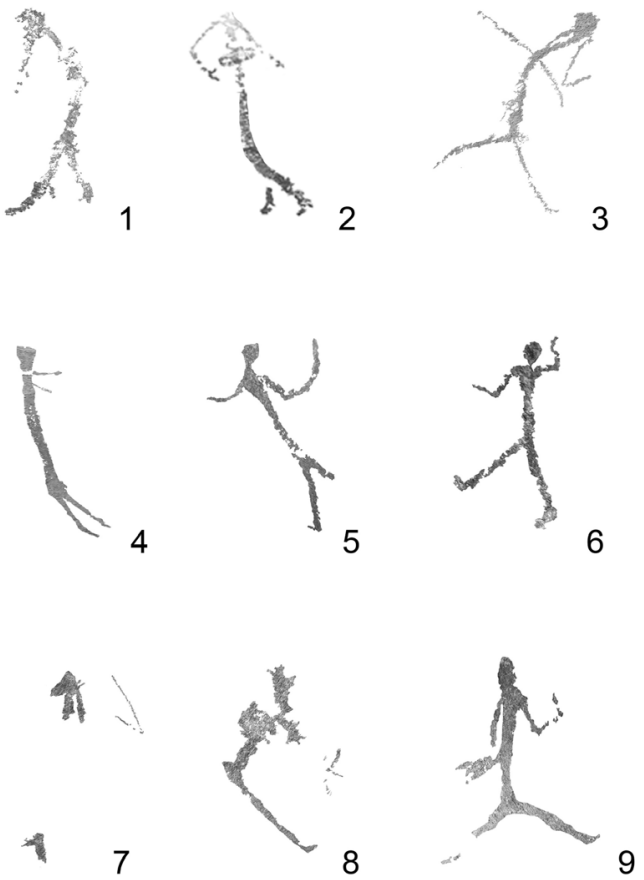

Figura 7. Paralelos para los antropomorfos del panel 2. esquemáticos, se encuentran en los conjuntos de las Cabras Blancas y abrigo de las Figuras Diversas, si bien en otros abrigos, como el de Doña Clotilde, aparecen representaciones que responden a la citada conformación básica. Incluso en aquellos casos en los que las figuraciones humanas no presentan una estilización tan marcada, o más bien rasgos próximos al esquematismo, se evidencia un gusto meridiano por la falta de detalles anatómicos y sencillez de trazo. Es el caso del grupo de arqueros del abrigo de los Toros del Prado del Navazo.

Independientemente de la coloración empleada en su confección, en negro-grisáceo (Hoya de Navarejos III, Figuras Diversas) o en blanco (Cabras Blancas, Prado del Navazo), en estos ejemplos destaca la estilización y movimiento de los motivos, participando de forma activa en escenas, aspecto que contrasta abiertamente con la actitud de las representaciones zoomorfas. Sin embargo, es posible apreciar diferencias en los conjuntos de manera que en unos se documentan escenas de ejecución sincrónica (Cabras Blancas) mientras que en otros parece tratarse de adiciones (abrigo de las Figuras Diversas, Toros del Prado del Navazo y quizá también Hoya de Navarejos III).

Con respecto al cromatismo, resulta muy significativa la diferenciación cromática evi- 
denciada entre los dos paneles, empleándose un único color, o variedad tonal del mismo, en cada panel. Así, en el exterior tan sólo aparece representado el color blanco, aspecto que relaciona todavía más estas figuraciones con las de los abrigos de Cocinilla del Obispo, Prado del Navazo y Ceja de Piezarrodilla. Por el contrario, en el panel 2 (interior) sólo se empleó el color negro o variedad tonal del mismo, pudiéndose definir alguna representación como grisácea.

No se ha podido identificar ningún motivo de color rojo, al margen de las pequeñas manchas informes en la zona NW de la cámara ya referidas con anterioridad.

\section{Sobre terminologías, clasificaciones y ordenaciones}

En general, se ha tratado de establecer una evolución lineal para el arte levantino, contemplando la existencia de fases evolutivas diacrónicas para el mismo que ayudaran a organizar un, cada vez más, vasto número de conjuntos y motivos diferentes, definidos con criterios "no siempre bien explícitos y con algunas contradicciones internas" (Hernández 2009: 70). La dificultad en la clasificación de conjuntos y motivos dentro de este denominado arte levantino ha llevado a definir algún elemento como "no solamente ambiguo, sino peligrosamente erróneo" (Beltrán 1999: 31), subrayando, con buen criterio, que "el naturalismo no es sinónimo de arte levantino" (Villaverde et al. 2012: 100). Junto a ideas como las referidas, que apuntan la necesidad de repensar en global el propio término de arte levantino, en los últimos años han aparecido algunas propuestas, nuevas lecturas para motivos rupestres conocidos y nuevos hallazgos, a los que nos referimos más adelante, que determinan la enorme complejidad que se encierra en un solo término.

Conjuntos como el analizado de Hoya de Navarejos III, además de las puntualizaciones inmediatamente referidas y aseveraciones que hablan de "manifestaciones muy diferentes desde el punto de vista formal y temático" (Villaverde et al. 2012: 88), hacen que, necesariamente, nos planteemos si lo que consideramos arte levantino puede ser entendido como un estilo "homogéneo" y de desarrollo lineal, como mínimo para la zona de distribución más interior, especialmente para la Sierra de Albarracín.
Son numerosos los intentos de clasificación y ordenación cronológica relativa ${ }^{4}$. Sin embargo, casi siempre se ha considerado que toda representación de tendencia naturalista y de cronología postpaleolítica debía ser englobada dentro del amplio término de "arte levantino", tratando de encontrar acomodo, en ocasiones forzado ${ }^{5}$, para cada motivo en alguna de las fases de desarrollo planteadas para el mismo. La propia inercia generada por la idea de que todo lo naturalista postpaleolítico debía ser, por fuerza, considerado como levantino, ha llevado a convertirlo en un concepto ${ }^{6}$ en el que se llegan a incluir manifestaciones muy heterogéneas.

Que la problemática terminológica para el arte postpaleolítico es un hecho se ha destacado en diversos trabajos (Beltrán 1999; Viñas y Saucedo 2000; Domingo 2005; López 2007; Martínez Bea 2005; Hernández 2009; Bueno 2009; Villaverde et al. 2012) y se aprecia en la multiplicidad de términos empleados para definir esencialmente lo mismo, o con pequeñas variaciones, que nos permiten, a los investigadores, proponer clasificaciones personalizadas.

El de la asignación crono-cultural, siquiera relativa, de cada una de las fases es una problemática añadida a la de la propia definición estilística y que no ha hecho más que ahondar en presupuestos fijistas. En este sentido, las superposiciones de motivos pertenecientes a diferentes estilos -levantino/esquemático; esquemático/levantino 7 - han propiciado un caldo de cultivo óptimo al emplearse como elemento de apoyo para un posicionamiento cronológico y cultural u otro en relación al origen del arte levantino.

No se ha podido formular una solución derivada de estas propuestas o de otras que plantean, como forma de superar esta problemática, el reenfoque de los estudios sobre arte postpaleolítico hacia determinados campos de investigación, como la Arqueología del Paisaje en unos casos o hacia cuestiones como la técnica de realización en otros, reduccionismo a partir del cual se trata de definir todo un ciclo artístico.

Naturalmente, en ninguno de estos casos se encuentra solución a una cuestión cuya problemática no radica sólo en nuevas perspectivas de análisis de los conjuntos rupestres sino también, y sobre todo, en la propia definición del cuerpo teórico y terminológico empleados por la tradición investigadora. 
No consideramos factible establecer una teoría unificada para el arte levantino, toda vez que éste, como creemos, no parece ser sólo uno. Todas esas aparentes incongruencias citadas se resolverían, automáticamente, al considerar la posibilidad de que existan diversos ciclos de tendencia naturalista, propios de momentos y grupos culturales diferentes postpaleolíticos y que no necesariamente deben estar relacionados de algún modo.

Debemos tener en cuenta que el gusto por lo naturalista no se pierde en momentos recientes de la Prehistoria e incluso protohistóricos, tal y como se ha apuntado para algunos abrigos del núcleo rupestre de la Sierra de Albarracín (Cruz Berrocal 2004 y 2005; Martínez Bea 2005 y 2008), o del Maestrazgo turolense, como en La Vacada (Martínez Bea 2004; Olmos 2005) o en el recientemente descubierto "guerrero de Mosqueruela" (Lorrio y Royo 2013), cuyos rasgos estilísticos se aproximan mucho más al naturalismo levantino que a otra cosa, así como la reinterpretación de determinadas agrupaciones o motivos, como el jinete del abrigo X del Cingle de Mola Remigia (Castellón), bien entrado tradicionalmente en el debate de su asignación crono-cultural. Como ya se ha destacado para otros contextos, la asociación del pasado con el presente en las panoplias gráficas postpaleolíticas tendría continuidad a lo largo de toda la Prehistoria (Bueno 2009; Bueno et al. 2009: 142), aspecto que pensamos resulta igualmente aplicable para el caso que nos ocupa.

Asimismo, se podría considerar que el uso de la tendencia naturalista en la decoración se puede documentar igualmente en otros ejemplos, no siempre bien ponderados, como en las cerámicas ibéricas. Por otra parte, más cercanos estilísticamente al arte levantino que la mayoría de los paralelos en cerámicas impresas $^{9}$ citados, tradicionalmente, de forma recurrente.

El arte levantino es un ente complejo, preñado de conceptos terminológicos e ideas preconcebidas que, con el paso del tiempo y con los nuevos descubrimientos, sólo pueden conducir a enmarañar todavía más su propia definición.

Consideramos que seguir hablando de arte levantino entendido como un ciclo artístico único, aun con diferentes fases de desarrollo, para toda el área de distribución definida podría resultar erróneo. Es manifiesta la diversidad temática, estilística, de características formales y convencionalismos existentes entre, por ejemplo, los conjuntos levantinos septentrionales y los meridionales (Beltrán 1999: 34). Una realidad divergente que se ha tratado de explicar como parte de un todo global cuyas diferencias estarían en función o bien de fases evolutivas de un mismo estilo o como factores regionales o étnicos diferenciadores.

Sin poder descartar en absoluto estas explicaciones (que sin duda entran también en juego en el propio desarrollo de un arte naturalista concreto) la propia concepción del arte levantino como una especie de frontera conceptual ha impedido contemplar también la posibilidad de que podamos estar delante de diferentes procesos artísticos o ciclos propios de momentos cronológicos y filiaciones culturales diversas que tendrían como nexo de unión el gusto por la tendencia naturalista de las representaciones.

\section{Conclusiones}

El conjunto de Hoya de Navarejos III aparece como uno de los descubrimientos recientes más singulares de todo el panorama levantino de la Península Ibérica. Si bien le temática, estilo y cromatismo de las figuraciones que contiene encuentran paralelos en abrigos cercanos y bien conocidos, destacan determinados aspectos que le confieren un carácter único. Así, desde el punto de vista locacional, se pueden apreciar dos espacios bien diferenciados: un espacio interior, en el que se realizaron los dos paneles decorados; y un espacio exterior, que sirve de transición hacia la entrada del primero, que aparece abstraído del entorno con la delimitación del mismo mediante lajas hincadas en el suelo.

Atendiendo a cuestiones temático-estilísticas, destaca la representación de un bóvido (motivo 3 del panel 2) en el que sólo se plasmó el tercio superior del animal (cabeza, cuello y lomo) y una serie de trazos lineales de desarrollo diagonal descendente hacia la derecha y paralelos entre sí y ligeramente curvos que se pueden interpretar como las costillas del animal. Este tratamiento, que no consideramos factible atribuir a cuestiones de índole técnica (relleno interior listado), resulta único en todo el arte levantino.

De hecho, en el panel 2 tan sólo fueron representados zoomorfos incompletos: bien la cabeza o bien el cuerpo, pero ni un solo 
se ha conservado o fue confeccionado completo.

A tenor de todo lo expuesto, el conjunto de Hoya de Navarejos III se une a un grupo cada vez más numeroso de estaciones rupestres y referencias interpretativas que abren un nuevo horizonte para el arte levantino en general y de la Sierra de Albarracín en particular. Así, este yacimiento junto a la interpretación global del núcleo rupestre de Albarracín y a determinadas singularidades presentes en otros contextos geográficos ya referidas nos invitan a considerar la necesidad de tratar de establecer una redefinición terminológica y conceptual para el arte levantino. Pensamos que se debe valorar la complejidad del fenómeno artístico naturalista postpaleolítico asumiendo la posible existencia de ciclos artísticos naturalistas independientes entre sí que podrían o no coincidir en el tiempo, y obedecer a realidades culturales bien diferenciadas, pudiendo plantear la existencia efectiva de diferentes "núcleos originales y de difusión de ideas artísticas o de contenido a partir de ellos", como apuntara Beltrán (1999: 34 ), sin que, necesariamente, todos ellos formaran parte de un único ciclo artístico.

Contemplar esta idea exime al investigador de tener que posicionarse en alguno de los grupos que abogan por diferentes orígenes cronológicos y/o culturales del arte levantino. Nos libra de tratar de explicar incongruencias en el registro, de establecer clasificaciones (en ocasiones) forzadas para que todo tenga cabida dentro de una única idea, de incidir sistemáti- camente en una dicotomía levantino vs esquemático de la que resulta complicado salir.

Esta propuesta entraña, naturalmente, nuevas problemáticas y grandes retos. Por una parte, tratar de diferenciar verdaderos territorios de desarrollo de diferentes ciclos artísticos postpaleolíticos de tendencia naturalista. Por otra, establecer una nueva terminología y un uso generalizado de la misma que permita al investigador reconocer no tanto un amplio estilo artístico con multitud de variaciones internas (arte levantino hasta el momento), sino verdaderos ciclos naturalistas susceptibles de definir con mayor precisión rasgos formales, territorios y momentos de realización, un aspecto referido en algunos de los trabajos ya citados. En cualquier caso, no debemos perder de vista que la imitación aproximada de la realidad no tiene porqué implicar afinidad crono-cultural, que dos bóvidos naturalistas siempre se parecerán entre sí, advirtiéndonos además de los peligros de suponer un todo monolítico sujeto a las leyes de la evolución de cada grupo geográfico de abrigos pintados (Beltrán 1995: 315), en definitiva, que no todo lo que reluce tiene que ser arte levantino.

En esta línea, la generalización de las analíticas, la evolución de las técnicas de datación y la construcción de propuestas sólidas de ámbito regional aparecen como aquellos elementos que serán capaces de discriminar la complejidad del fenómeno artístico naturalista postpaleolítico que sólo somos capaces de inferir desde un punto de vista teórico.

\section{Notas}

3. Son relativamente numerosos los espacios cerrados, conocidos como "cerradas", cuya funcionalidad es la de servir de redil para el ganado. Algunos de estos cerramientos fueron construidos en abrigos con paneles decorados, como ocurre en los casos de Cerrada del Tío Jorge, Paridera de Tormón, Paridera de Bezas, Arroyo de Bezas II, Cerrada de Florentín, Hoya de Navarejos I y II y Barranco de la Casa Forestal V. Sin embargo, el concepto constructivo es totalmente diferente al que se observa en el conjunto que nos ocupa. En todos los casos anteriores se trata de verdaderos muros en piedra seca que llegan a sobrepasar los $1,8 \mathrm{~m}$ de altura y construidos con bloques de arenisca cuadrangulares normalmente de tamaño pequeño-medio. En ningún caso se constata la existencia de la delimitación de un espacio mediante lajas hincadas, cuya disposición y entidad difícilmente podrían haber cumplido una función de retención física del ganado. El espacio que se delimita en Hoya de Navarejos III es además una superficie totalmente expuesta a cielo abierto, no hay cornisa que permita cobijar o proteger al ganado, como sí ocurre en el resto de los casos referidos. Además, tras consultar con las gentes del lugar, pudimos constatar que nadie conocía nada semejante en el territorio. Por todo ello, concluimos que las lajas no podían contemplarse como un cerramiento para el ganado de cronología subactual, sino que su presencia debía entenderse en relación al conjunto rupestre y que su lectura simbólica podría estar relacionada con éste. Naturalmente, hasta que no se realicen los sondeos arqueológicos previstos en el yacimiento no se puede contar con otros datos que permitan relacionar panel pintado y delimitación del espacio. Sin embargo, y en el momento de investigación actual, las particularidades definidas para éste nos permiten descartar su naturaleza como un elemento constructivo subactual 
relacionado con el cerramiento del ganado y, por consiguiente, apoya más la opción de su consideración como delimitador simbólico del espacio.

4. Estas ordenaciones se han tratado de establecer, como mayor o menor éxito, desde el inicio mismo de los estudios sobre arte levantino, siendo algunas de las más recientes las de Domingo $(2005,2012)$, López (2007), Martínez Bea (2005), Martínez i Rubio (2011), Utrilla (2000), Utrilla y Martínez Bea (2007) o Villaverde et al. (2012).

5. Es el caso de las representaciones de las cuevas de Peña Rubia de Ceheguín. Los motivos, alejados formalmente de los rasgos que definen un arte esquemático que sería más acorde tradicionalmente con el contexto arqueológico en el que aparecen (interior de cuevas funerarias eneolíticas), llevan a los autores del estudio a definirlos como levantinos. Así, y una vez asumida esta asignación, y a pesar de que los propios autores expresan que "no existe la menor relación estilística de estas pinturas con las levantinas de la región murciana" (Beltrán y San Nicolás 1988: 43), la necesidad impuesta por su definición como arte levantino les lleva a buscar explicaciones en "una evolución a través de los tiempos", "perduración de elementos antiguos" o incluso como "degeneración del arte levantino clásico que evolucionaría en un círculo cerrado" (ibidem 1988: 43-45), asumiendo que el arte levantino se agotaría hacia el Eneolítico (ibídem 1988: 46).

6. Algo similar podría ocurrir con el arte esquemático. Si bien en este caso sí se ha reconocido la existencia de diferentes horizontes crono-culturales con una tradición artística esquemática, no es menos cierto que casi siempre se ha aludido a su naturaleza prehistórica, obviando la posibilidad de que determinados conjuntos o motivos dentro de éstos pudieran resultar históricos (Bea 2013; Bea et al. 2009) o incluso plenamente contemporáneos, como recientemente se ha destacado para el caso de los motivos piqueteados del Huerto de las Tajadas de Bezas (Bea y Angás 2013).

7. La Sarga: levantino sobre macroesquemático, aunque dependiendo del investigador la ordenación podría resultar la inversa. Tío Modesto: esquemático sobre levantino. Labarta: levantino sobre lineal-geométrico. Los Chaparros: levantino sobre lineal-geométrico...

8. El tratamiento de la representación, sobre todo, en las piernas resulta perfectamente asumible dentro de la definición para la figura humana levantina: buenas proporciones, modelado de la musculatura, representación de detalles de la vestimenta (faldellín hasta los muslos, algún tipo de elemento ornamental o protector en las pantorrillas). Son los elementos que porta (espada, vaina y escudo) y el tocado de cuernos de la cabeza lo que permite definir la figura como un verdadero guerrero celtíbero.

9. Basten citar el paralelo de árbol de la cerámica cardial de Cova de la Sarsa y su relación propuesta con la escena de vareo de La Sarga o las figuraciones impresas de cabra y ciervo de las de Cova de l’Or.

\section{Referencias bibliográficas}

Almagro, M. (1970): Manual de Historia Universal. Madrid.

Bader, M.; Bader, K.; Viñas, R. (1981): Noticia sobre una nueva estación de arte rupestre en Albarracín (Teruel). Cuadernos de Prehistoria y Arqueología Castellonense, 8: 307-309.

Barciela, V.; Martorell, X.; Molina, F.J. (2015): Arte rupestre prehistórico en la Serra de Segària (Alicante, España). Sobre rocas y huesos: las sociedades prehistóricas y sus manifestaciones plásticas ( ${ }^{\mathrm{a}}$.A. Medina; A.J. Romero; R.Mª Ruiz-Márquez; J.L. Sanchidrián, eds.), Fundación Cueva de Nerja y Universidad de Córdoba. Córdoba: 286-299.

Bea, M. (2013): En Arte rupestre esquemático pre-Histórico. Nueva interpretación de los carros de Remosillo (Olvena, Huesca). II Congreso Arte Rupestre Esquemático en la Península Ibérica, Vélez-Blanco: 243-252.

Bea, M. (2014): Novedades en torno al núcleo de arte levantino de la Sierra de Albarracín. El abrigo de Prao Medias (Tormón, Teruel). Saguntum, 46: 203-207. https://ojs.uv.es/index.php/saguntum/article/ view/3068.

Bea, M.; Angás, J. (2013): Reestudio de los conjuntos rupestres de las Tajadas de Bezas (Teruel). Cuadernos de Arte Rupestre, 6: 129-145.

Bea, M.; Angás, J. (2015): Las representaciones levantinas de bovinos de la Casa Forestal de Tormón (Teruel): Ceja de Piezarrodilla y Cerrada del Tío Jorge. Zephyrus, LXXV: 73-84. http://campus.usal. es/ revistas_trabajo/index.php/0514-7336/article/view/zephyrus2015757384.

Bea, M.; Domingo, R.; Uribe, P.; Reklaityte, I.; Fatás, L. (2009): Actuaciones arqueológicas en los abrigos de Roca dels Moros y Els Gascons (Cretas), Teruel) y de La Fenellosa (Beceite, Teruel). Salduie, 9: 393-418.

Beltrán, A. (1968): Arte rupestre levantino. Monografías Arqueológicas, IV. Universidad de Zaragoza, Zaragoza. 
Beltrán, A. (1987): Las pinturas en el interior de las Cueva de la Peña Rubia (Cehegín, Murcia). Historia, anécdota y estudio. Caesaraugusta, 64: 7-86.

Beltrán, A. (1993): Arte prehistórico en Aragón. IberCaja, Zaragoza.

Beltrán, A. (1995): Sobre el arte levantino, especialmente de Albarracín. Ideas generales para un debate. XXI Congreso Nacional de Arqueología, Zaragoza: 289-315.

Beltrán, A. (1997): Las pinturas prehistóricas de la Paridera de Tormón. Arqueología Aragonesa 1994: 45-49.

Beltrán, A. (1999): Cronología del arte levantino: cuestiones críticas. Cronología del arte rupestre levantino, Real Academia de Cultura Valenciana, Valencia: 7-41.

Beltrán, A.; San Nicolás, M. (1988): Las pinturas de las Cuevas de Peña Rubia. Institución Fernando el Católico, Zaragoza.

Bueno, P. (2008): Espacios decorados al aire libre del occidente peninsular. Territorios tradicionales de cazadores-recolectores y de productores. Arte prehistórico al aire libre en el Sur de Europa (R. de Balbín, ed.), Junta de Castilla y León, Salamanca: 321-347.

Bueno, P.; de Balbín, R.; Barroso, R. (2009): Pintura megalítica en Aadalucía. Estudios de prehistoria y Arqueología en Homenaje a Pilar Acosta Martinez (R. Cruz-Auñón y E. Ferrer, coords.), Universidad de Sevilla, Sevilla: 141-169.

Burillo, F.; Picazo, J.V. (1981): Nuevo hallazgo de pinturas levantinas en el barranco del Hocino de Chornas. Obón (Teruel). Kalathos, 1: 75-91.

Collado, O.; Cotino, F.; Ibáñez, R.; Nieto, E. (1991-1992): Revisión del abrigo de las Cabras Blancas. Kálathos, 11-12: 25-42.

Cruz Berrocal, $M^{\mathrm{a}}$. (2004): La investigación del arte rupestre desde la Geografía: la pintura neolítica del ámbito mediterráneo de la Península Ibérica. Trabajos de Prehistoria, 61 (2): 41-62. http://tp.revistas. csic.es/index.php/tp/article/view/42/42.

Cruz Berrocal, Mª (2005): Los sistemas de arte rupestre en entornos locales. Arte Rupestre en la España Mediterránea (M.S. Hernández y J.A. Soler, eds.), Alicante: 161-168.

Domingo, I. (2005): Técnica y evolución de la figura en el arte rupestre levantino. Hacia una definición actualizada del concepto de estilo: validez y limitaciones. Servei de Publicacions. Universidad de Valencia, Valencia.

Domingo, I. (2012): Figura humana, técnicas y territorios: hacia una redefinición técnica del arte rupestre levantino". El problema levantino (J.J. García; H. Collado; G. Nash, eds.), Archaeolingua. BudapestCáceres: 117-144.

Hernández, M.S. (2009): Arte rupestre postpaleolítico en el Arco Mediterráneo de la Península Ibérica. Balance de 10 años de descubrimientos y estudios. El arte rupestre del Arco Mediterráneo de la Península Ibérica. 10 años en la lista del Patrimonio Mundial de la UNESCO (J.A. Mira; R. Martínez y C. Matamoros, eds.), Generalitat Valenciana, Valencia: 59-79.

López, E. (2007): Análisis interno del arte levantino: la composición y el espacio a partir de la sistematización del núcleo Valltorta-Gasulla. Servei de Publicacions. Universidad de Valencia, Valencia.

Lorrio, A.; Royo, J.I. (2013): El guerrero celtibérico de Mosqueruela (Teruel): una pintura rupestre excepcional de la Edad del Hierro en el Alto Maestrazgo turolense. Antiquitas, 25: 85-107.

Martínez Bea, M. (2004): Un arte no tan levantino. Perduración ritual de los abrigos pintados: el ejemplo de La Vacada (Castellote, Teruel). Trabajos de Prehistoria, 61 (2): 111-125. http://tp.revistas.csic.es/ index.php/tp/article/view/46/46.

Martínez Bea, M. (2005): Variabilidad estilistica y distribución territorial del arte rupestre levantino en Aragón: el ejemplo de La Vacada (Castellote, Teruel). Universidad de Zaragoza, Zaragoza.

Martínez Bea, M. (2008): Arte rupestre de Albarracín: la excepcionalidad de un conjunto interior. IV Congreso de Neolítico Peninsular (M.S. Hernández; J.A. Soler y J.A. López, eds.), Museo Arqueológico Provincial de Alicante (MARQ), Alicante: 141-148.

Martínez i Rubio, T. (2011): Evolució i pautes de localització de l'art rupestre postpaleolític en Millares (València) i el seu entorn geogràfic comarcal. Aproximació al territori des de l'art. Servei de Publicacions. Universidad de Valencia, Valencia.

Obermaier, H. (1927): Nuevas pinturas rupestres descubiertas en los alrededores de Tormón (Teruel). Investigación y Progreso, I (1): 1-2.

Obermaier, H.; Breuil, H. (1927): Pinturas rupestres de los alrededores de Tormón (Teruel). Boletín de la Real Academia de Historia, 90: 511-531.

Olmos, R. (2005): Iconografía celtibérica. Celtíberos. Tras la estela de Numancia (A. Jimeno, dir.), Junta de Castilla y León, Soria: 253-260.

Piñón, F. (1981): Superposiciones y repintados en la Serranía de Albarracín: elementos para el establecimiento de una cronología relativa. Altamira Symposium, Madrid: 411-426.

Piñón, F. (1982): Las pinturas rupestres de Albarracín (Teruel). Monografías del Centro de Investigación y Museo de Altamira, 6. Ministerio de Cultura, Santander. 
Ripoll, E. (1968): Cuestiones en torno a la cronología del arte rupestre postpaleolítico en la península Ibérica. Simposio Internacional de Arte Rupestre, Barcelona: 165-192.

San Nicolás, M. (ed.) (2009): El conjunto prehistórico y de arte rupestre de El Milano. Mula, Murcia. Monografías CEPAR, 1, Murcia.

Utrilla, P. (2000): Arte rupestre en Aragón. Colección CAI100, 56, Zaragoza.

Utrilla, P.; Martínez Bea, M. (2007): La figura humana en el arte levantino aragonés. Cuadernos de Arte Rupestre, 4: 163-205.

Villaverde, V.; Martínez, R.; Guillem, P.; López, E.; Domingo, I. (2012): ¿Qué entendemos por arte levantino?. El problema levantino (J.J. García; H. Collado; G. Nash, eds.), Archaeolingua, Budapest-Cáceres: $81-115$.

Viñas, R.; Saucedo, E. (2000): Los cérvidos en el arte rupestre postpaleolítico. Quadernos de Prehistoria y Arqueología Castellonenses, 21: 53-68. 